\title{
Soya isoflavone consumption in relation to carotid intima-media thickness in Chinese equol excretors aged $40-65$ years
}

\author{
Yun Cai ${ }^{1} \dagger$, Kaiping Guo ${ }^{1} \dagger$, Chaogang Chen ${ }^{2}$, Ping Wang ${ }^{1}$, Bo Zhang ${ }^{1}$, Quan Zhou ${ }^{1}$, Fang Mei $^{1}$ and \\ Yixiang $\mathrm{Su}^{1 *}$ \\ ${ }^{1}$ Faculty of Nutrition, School of Public Health, Sun Yat-Sen University, Guangzhou 510080, People's Republic of China \\ ${ }^{2}$ Department of Clinical Nutrition, The Second Affiliated Hospital of Sun Yat-Sen University, Guangzhou, People's Republic of \\ China
}

(Submitted 16 March 2011 - Final revision received 7 December 2011 - Accepted 7 December 2011 - First published online 29 February 2012)

\begin{abstract}
Previous studies have suggested that the daidzein metabolite equol rather than daidzein itself contributes to the beneficial effect of soya foods in the prevention of CVD. The aim of the present study is to examine the proportion of equol excretion in Chinese adults and compare plasma lipids and carotid artery intima-media thickness (IMT) between equol excretors and non-excretors, and to evaluate the effect of soya isoflavone intakes on serum lipids and IMT in either equol excretors or non-excretors. Subjects ( $n$ 572; women $n$ 362, men $n$ 210) were recruited for the present study. An overnight urine sample was provided by each subject on their usual diet to quantify urinary concentrations of daidzein and equol. Far-wall IMT was determined by B-mode ultrasound in the right carotid at two sites, carotid bulb (CB-IMT) and common carotid artery (CCA-IMT), and fasting serum lipids were measured. Habitual dietary intakes were estimated with a FFQ, and soya isoflavone intake derived from the FFQ was assessed. Of the 572 subjects, the proportion of equol excretors on their usual diet was $25.0 \%$ ( $n$ 143). Compared with non-excretors, equol excretors showed significantly lower serum TAG (-38.2 (95\% CI $-70 \cdot 4,-5.9) \%, P=0.012)$ and CCA-IMT $(-4.9(95 \% \mathrm{CI}-9.7,-0.3) \%, P=0.033)$. Equol excretors with higher daily isoflavone intakes $(-5.4 \mathrm{mg} / \mathrm{d})$ had significantly lower IMT $(-16.2 \%, P=0.035)$ and tended to have higher HDL-cholesterol $(P=0.055)$ than did those with lower daily isoflavone intakes $(1.5 \mathrm{mg} / \mathrm{d})$, while no association was observed between soya isoflavone intakes and serum lipids or IMT in non-excretors. In conclusion, the benefits of soya isoflavones in preventing CVD may be apparent among equol excretors only.
\end{abstract}

Key words: Isoflavones: Equol: Blood lipids: Carotid artery intima-media thickness

Soya isoflavones, mainly daidzein and genistein, have a similar chemical structure to that of oestrogens ${ }^{(1)}$, and consequently bind to oestrogen receptors to exert either oestrogenic or anti${\text { oestrogenic } \text { activity }^{(2)} \text {. Many studies }}^{(3,4)}$ have indicated that higher soya intake is associated with a lower incidence of CVD.

The bioactive effects of isoflavones with or without soya protein have been fully studied. In three recent meta-analyses, it has been confirmed that soya protein with isoflavones, when compared with other proteins, had clinically important favourable effects on LDL-cholesterol and other CVD risk factors $^{(5-7)}$, whereas the results of studies comparing soya protein that did or did not contain isoflavones or comparing isoflavones in pill form with placebo were inconsistent ${ }^{(7,8)}$. One possible explanation for complexity in the responsiveness to isoflavones is due to the differences in the metabolism of isoflavones among individuals, specifically the variance in equol-synthesising capacity ${ }^{(9)}$.
Equol is produced by the intestinal bacterial flora from daidzein in approximately one-third to one-half of human subjects $^{(1)}$. Previous studies have shown a substantial individual difference in the ability to produce equol ${ }^{(9)}$, and Asian populations have a higher equol-producer prevalence ${ }^{(10)}$. Equol is believed to be the active form of soya isoflavones and has a stronger oestrogen-like activity than daidzein. Thus, the equol phenotype may modify the protection value of soya isoflavones against hormone-dependent diseases and affect the lipid profile and vascular health ${ }^{(11,12)}$.

Therefore, the present study aims to investigate the prevalence of equol excretion under the usual diet in southern Chinese adults who are usual soya consumers, and to assess the association between the ability of equol excretion and lipid profiles and intima-media thickness (IMT), and to evaluate the relationship between isoflavone intakes and lipid profiles and IMT in either equol excretors or non-excretors.

Abbreviations: CCA, common carotid artery; IMT, intima-media thickness.

*Corresponding author: Y. Su, email suyx@mail.sysu.edu.cn

†These authors contributed equally to this work. 


\section{Experimental methods}

\section{Subjects and study procedure}

A total of 600 community-based volunteers aged 40-65 years were recruited in Guangzhou, China by poster and telephone. After initially screening for their eligibility using a short questionnaire, which recorded sex, age and exclusion criteria, potential subjects were then invited to the First or the Second Affiliated Hospital of Sun Yat-sen University, Guangzhou, China. Staff with relevant knowledge in medical sciences screened the subject for eligibility via a face-to-face interview to determine whether they met the inclusion and exclusion criteria. Subjects were excluded if they had a history of diseases, which may affect personal lifestyle, such as CVD, diabetes, cancer, dyslipidaemia, or had recently (3 months) used drugs or healthy products affecting blood lipids. Carotid IMT was measured unilaterally (right) at the carotid bulb and common carotid artery (CCA-IMT, $20 \mathrm{~mm}$ proximal to the bulb) at the far wall of the artery using Technos MPX DU8 (Esaote) with the frequency of the probe set at 10.0 MHz. All subjects were examined in a supine position after they had rested for $10 \mathrm{~min}$. B-mode images at the diastolic phase of the cardiac cycle were recorded by a single trained technician who was kept unaware of the subjects' backgrounds. On a longitudinal, two-dimensional ultrasound image of the carotid artery, the far walls of the carotid artery were displayed as two bright white echogenic lines separated by a hypoechogenic space. The distance between the leading edge of the first bright line and the leading edge of the second bright line indicates the IMT. Plaque thickness was avoided in IMT measurements.

The study was conducted according to the guidelines laid down in the Declaration of Helsinki and all procedures were approved by the Medical Ethics Committee of Sun Yat-sen University. All participants signed the written informed consent form.

Information on sociodemographic data, medication and health history, lifestyles, socio-economic status and physical condition was obtained from a structured questionnaire. Information about habitual dietary intakes within the past 12 months before the investigating day was estimated using a FFQ designed for Chinese populations via a face-to-face interview by trained investigators. The first urine passed in the morning was provided by each subject on their usual diet to quantify urinary concentrations of isoflavones ${ }^{(13,14)}$, and $10 \mathrm{ml}$ of the samples were stored at $-80^{\circ} \mathrm{C}$ before analysis. Height, weight, waist and hip circumferences and blood pressure were measured. Fasting blood samples $(10 \mathrm{ml})$ were collected for blood lipid analysis.

Of the 600 subjects, twenty-eight subjects (ten men and eighteen women) were excluded for failure to return urine collection. A total of 572 participants successfully completed the study and were included in the data analysis.

\section{Dietary information}

Food models were used to help subjects in the quantification of their diet.
The FFQ includes 118 foods and food groups that cover the most commonly consumed foods in Guangzhou, China. The FFQ includes the following soya and isoflavone-rich food items or groups: soya milk, tofu, fried tofu, dried soyabeans, mung beans, soyabean sprouts, fresh beans and peanuts. For each food item or food group, subjects were asked about the frequency (daily, weekly, monthly, annually or never) and the amount in Liang (Liang $=50 \mathrm{~g}$ ) per unit of time. The percentage of energy from fat for each subject was calculated according to the FFQ. The soya isoflavone intake levels derived from the FFQ were assessed using the Chinese Food Composition Table. Total isoflavone intake was calculated using the following formula:

Total isoflavone intake $=\Sigma($ amount of soya food from the FFQ $\times$ proportion of edible part $x$ isoflavone amount of each soya food).

\section{Measurements}

Height, weight, waist and hip circumferences and blood pressure were measured on the day of urine collection. A $12 \mathrm{~h}$ fasting venous blood sample was collected in vacuum tubes containing EDTA. Plasma was separated after centrifugation at $1500 \mathrm{~g}$ for $15 \mathrm{~min}$ at $4^{\circ} \mathrm{C}$ within $2 \mathrm{~h}$ and was stored at $-80^{\circ} \mathrm{C}$ until analysis. Plasma lipids were measured using a Hitachi 7600-010 automatic analyser (Hitachi). The CV for lipid measurements were $2 \cdot 17 \%$ (at 5.03 mm-total cholesterol), $2 \cdot 86 \%$ (at $1.14 \mathrm{~mm}$-TAG), $3.47 \%$ (at $1.70 \mathrm{~mm}$-HDL-cholesterol) and $4.67 \%$ (at $2.65 \mathrm{~mm}$-LDL-cholesterol). Biochemical assays were performed by two technicians who were not involved in the questionnaire interview.

Soya isoflavone metabolites in the first urine passed in the morning were assayed by HPLC with UV detection. In brief, urine samples were extracted by ethyl acetate $(5 \mathrm{ml} \times 2)$ after deconjugation by $\beta$-glucuronidase/sulphatase. After evaporated to dryness under $\mathrm{N}_{2}$ at $40^{\circ} \mathrm{C}$, the extract was reconstituted in a mobile-phase solution $(1 \mathrm{ml})$ for analysis. The HPLC system consisted of a C18 stationary-phase extraction ( $5 \mu \mathrm{m}, 4.6 \Phi \times 250 \mathrm{~mm}$ ) column, and separation of isoflavones was achieved with the mobile phase at a flow rate of $1.0 \mathrm{ml} /$ min with the following gradient elution: A - acetic diethyl ether-methanol-0.05\% phosphate buffer (1:10:40, by vol.); $\mathrm{B}$ - acetic diethyl ether-methanol (1:49, v/v); 0.0-35.0 min, $0-35 \% \quad \mathrm{~B} ; \quad 35 \cdot 0-45 \cdot 0 \mathrm{~min}, \quad 35-35 \% \mathrm{~B} ; \quad 45 \cdot 0-50 \cdot 0 \mathrm{~min}$, $35-40 \% \quad \mathrm{~B} ; \quad 50 \cdot 0-55.0 \mathrm{~min}, \quad 40-70 \% \mathrm{~B} ; \quad 55 \cdot 0-60.0 \mathrm{~min}$, $70-0 \% \mathrm{~B} ; 60 \cdot 0-70 \cdot 0 \mathrm{~min}, 0-0 \% \mathrm{~B}$. Isoflavones were detected from their UV absorbance at 254 and $280 \mathrm{~nm}$. The $\mathrm{CV}$ for daidzein and equol measurements were $<5 \%$.

Subjects with urinary equol:daidzein ratios $>0.018$ were defined as equol excretors ${ }^{(15)}$.

\section{Data analysis}

Data were checked for normality, and skewed parameters were log transformed before statistical analysis when possible. Data that were normally distributed either before or after log 
transformation were presented as means and standard deviations. Isoflavone intakes were categorised by the quartile distribution of the study population in the analysis. Differences between the two groups were assessed by Student's $t$ test. Comparisons of variables between different quartiles of isoflavone intakes were performed with one-way ANOVA with post hoc Bonferroni's correction for multiple comparisons and the test for a linear trend. ANCOVA was used to compare the mean differences in blood lipids and IMT after adjustment for other potential confounding factors. The level of significance was set at $P<0.05$. All analyses were conducted using SPSS for Windows (version 11.5; SPSS, Inc.).

\section{Results}

Of the 572 subjects, 76.4\% ( $n$ 437) had detectable daidzein, $25 \cdot 2 \%$ ( $n$ 144) had detectable urinary equol and 25\% ( $n$ 143) were defined as equol excretors. The proportions of equol excretors were $26.2 \%$ in men and $24.4 \%$ in women, with no statistical difference between men and women $(P>0.05)$.

There was no significant association between equolmetabolising phenotype in sociodemographic factors of subjects, such as age, sex, socio-economic status and education
(Table 1). Subjects with different equol-metabolising phenotypes had no significant differences in the dietary intakes of soya isoflavones, energy and fat.

Equol excretors showed lower serum TAG $(-38.2(95 \%$ CI $-70 \cdot 4,-5.9) \%, P=0.012)$ and CCA-IMT $(-4.9(95 \%$ CI $-9.7,-0.3) \%, P=0.033)$ compared with non-excretors. No significant differences in BMI, waist:hip ratio and blood pressure were observed between equol excretors and nonexcretors (Table 1).

Subjects were categorised into four quartiles by soya isoflavone intake. For both equol excretors and non-excretors, subjects in the four quartiles of daily isoflavones had significant differences in the intakes of daily energy and fat (\% energy, $P<0.05$ for both). There were no significant differences in BMI, waist:hip ratio, and systolic and diastolic blood pressure with increasing quartiles of isoflavones $(P>0.05$ for all; Table 2).

After adjustment for age, sex, daily energy intake and fat (\% energy), there was a significant linear trend in serum HDLcholesterol concentration with increasing quartiles of isoflavones $(P=0 \cdot 017$; Table 3$)$ in equol excretors. Also, subjects in the subpopulation with more daily isoflavone intakes tended to have higher HDL-cholesterol concentration

Table 1. Socio-economic, dietary clinical, and lipid profile of the participants by equol status (Mean values and standard deviations)

\begin{tabular}{|c|c|c|c|c|c|}
\hline & \multicolumn{2}{|c|}{$\begin{array}{l}\text { Equol excretors } \\
\qquad(n 143)\end{array}$} & \multicolumn{2}{|c|}{$\begin{array}{l}\text { Non-equol excretors } \\
\qquad(n 429)\end{array}$} & \multirow[b]{2}{*}{$P^{\star}$} \\
\hline & Mean & SD & Mean & SD & \\
\hline \multicolumn{6}{|l|}{ Demographic } \\
\hline Age (years) & $52 \cdot 6$ & 5.4 & $52 \cdot 8$ & $5 \cdot 3$ & 0.623 \\
\hline Sex (female, \%) & \multicolumn{2}{|c|}{61.5} & \multicolumn{2}{|c|}{63.8} & 0.689 \\
\hline BMI $\left(\mathrm{kg} / \mathrm{m}^{2}\right)$ & $22 \cdot 6$ & 3.4 & 22.9 & 3.2 & 0.330 \\
\hline WHR & 0.87 & 0.07 & 0.87 & 0.07 & 0.966 \\
\hline Education (\%) & & & & & 0.786 \\
\hline None or primary & \multicolumn{2}{|c|}{$13 \cdot 4$} & \multicolumn{2}{|c|}{$13 \cdot 3$} & \\
\hline Junior school & \multicolumn{2}{|c|}{33.8} & \multicolumn{2}{|c|}{33.5} & \\
\hline High school & \multirow{2}{*}{\multicolumn{2}{|c|}{$\begin{array}{r}48.6 \\
4.2\end{array}$}} & \multicolumn{2}{|c|}{$46 \cdot 6$} & \\
\hline College or above & & & \multicolumn{2}{|c|}{6.6} & \\
\hline Monthly income (\%) & & & & & 0.565 \\
\hline 500 & \multicolumn{2}{|c|}{$17 \cdot 4$} & \multicolumn{2}{|c|}{17.5} & \\
\hline $500-1000$ & \multicolumn{2}{|c|}{$31 \cdot 2$} & \multicolumn{2}{|c|}{$25 \cdot 2$} & \\
\hline $1001-2000$ & \multirow{2}{*}{\multicolumn{2}{|c|}{$\begin{array}{l}38.4 \\
13.0\end{array}$}} & \multicolumn{2}{|c|}{$42 \cdot 2$} & \\
\hline 2000 & & & & & \\
\hline \multicolumn{6}{|l|}{ Dietary } \\
\hline Soya isoflavone intake $(\mathrm{mg} / \mathrm{d})$ & 13.7 & $16 \cdot 7$ & $13 \cdot 3$ & $32 \cdot 8$ & 0.881 \\
\hline Energy $(\mathrm{kJ} / \mathrm{d})$ & 10113 & 4577 & 10000 & 5184 & 0.818 \\
\hline Fat $(\mathrm{g} / \mathrm{d})$ & $98 \cdot 3$ & 81.4 & 95.1 & $94 \cdot 2$ & 0.713 \\
\hline Fat (\% energy) & $35 \cdot 1$ & $12 \cdot 2$ & $34 \cdot 1$ & $12 \cdot 3$ & 0.419 \\
\hline \multicolumn{6}{|l|}{ Clinical } \\
\hline $\mathrm{SBP}(\mathrm{mmHg})$ & 123.4 & $19 \cdot 6$ & $122 \cdot 3$ & $17 \cdot 3$ & 0.603 \\
\hline $\mathrm{DBP}(\mathrm{mmHg})$ & $79 \cdot 7$ & 9.5 & $80 \cdot 3$ & $10 \cdot 2$ & 0.602 \\
\hline $\mathrm{TC}(\mathrm{mmol} / \mathrm{l})$ & 5.53 & 0.86 & $5 \cdot 63$ & 0.95 & 0.362 \\
\hline $\mathrm{HDL}-\mathrm{C}(\mathrm{mmol} / \mathrm{l})$ & 1.54 & 0.47 & 1.51 & 0.38 & 0.520 \\
\hline LDL-C (mmol/l) & 3.35 & 0.73 & 3.32 & 0.77 & 0.793 \\
\hline $\mathrm{TAG}(\mathrm{mmol} / \mathrm{l}) \dagger$ & 0.08 & 0.20 & 0.15 & 0.25 & 0.012 \\
\hline CB-IMT (mm) & 0.99 & 0.28 & 1.03 & 0.36 & 0.279 \\
\hline CCA-IMT (mm) & 0.54 & 0.13 & 0.62 & 0.34 & 0.033 \\
\hline
\end{tabular}

WHR, waist:hip ratio; SBP, systolic blood pressure; DBP, diastolic blood pressure; TC, total cholesterol; HDL-C, HDL-cholesterol; LDL-C, LDL-cholesterol; CB-IMT, intima-media thickness at the carotid bulb; CCA-IMT, intima-media thickness at the common carotid artery.

${ }^{*}$ Student's $t$ test for continuous variables and $\chi^{2}$ tests for proportion.

† Log transformed. 
Table 2. Dietary and anthropometric characteristics across quartiles of soya isoflavone intakes in equol excretors or non-excretors* (Mean values and standard deviations)

\begin{tabular}{|c|c|c|c|c|c|c|c|c|c|}
\hline & \multicolumn{8}{|c|}{ Soya isoflavone intakes } & \multirow[b]{3}{*}{$P$} \\
\hline & \multicolumn{2}{|c|}{1 (low) } & \multicolumn{2}{|c|}{2} & \multicolumn{2}{|c|}{3} & \multicolumn{2}{|c|}{4 (high) } & \\
\hline & Mean & SD & Mean & SD & Mean & SD & Mean & SD & \\
\hline \multicolumn{10}{|l|}{ Equol excretors } \\
\hline$n$ & 35 & & 36 & & 36 & & 36 & & \\
\hline Sex (male/female) & $14 / 22$ & & $14 / 22$ & & $14 / 22$ & & $13 / 22$ & & \\
\hline Isoflavones (mg/d) & 1.5 & $1 \cdot 1$ & 5.4 & $1 \cdot 1$ & $12 \cdot 1$ & $3 \cdot 3$ & $35 \cdot 6$ & $19 \cdot 9$ & \\
\hline Range & \multicolumn{2}{|c|}{$0.02-4.48$} & \multicolumn{2}{|c|}{$1.24-8.37$} & \multicolumn{2}{|c|}{$6 \cdot 33-21 \cdot 11$} & \multicolumn{2}{|c|}{$15 \cdot 04-119.50$} & \\
\hline Energy $(\mathrm{kJ} / \mathrm{d})$ & 8368 & 2347 & 9167 & 4460 & 9468 & 3594 & 10113 & 4577 & 0.000 \\
\hline Fat (\% energy) & 33.2 & $10 \cdot 2$ & $36 \cdot 2$ & $11 \cdot 3$ & $30 \cdot 2$ & $12 \cdot 9$ & $35 \cdot 1$ & $12 \cdot 2$ & 0.002 \\
\hline BMI $\left(\mathrm{kg} / \mathrm{m}^{2}\right)$ & $23 \cdot 8$ & $3 \cdot 2$ & $23 \cdot 2$ & $3 \cdot 0$ & $23 \cdot 1$ & $3 \cdot 8$ & $22 \cdot 6$ & 4.3 & 0.554 \\
\hline WHR & 0.89 & 0.07 & 0.88 & 0.06 & 0.87 & 0.06 & 0.87 & 0.06 & 0.260 \\
\hline $\mathrm{SBP}(\mathrm{mmHg})$ & $129 \cdot 8$ & $17 \cdot 9$ & $123 \cdot 1$ & $17 \cdot 2$ & $124 \cdot 7$ & $18 \cdot 4$ & $128 \cdot 4$ & $24 \cdot 3$ & 0.433 \\
\hline $\mathrm{DBP}(\mathrm{mmHg})$ & $82 \cdot 7$ & $8 \cdot 0$ & 79.6 & $9 \cdot 8$ & 80.4 & $10 \cdot 3$ & $80 \cdot 8$ & $9 \cdot 8$ & 0.568 \\
\hline \multicolumn{10}{|l|}{ Non-excretors } \\
\hline$n$ & 107 & & 107 & & 108 & & 107 & & \\
\hline Sex (male/female) & $39 / 69$ & & $39 / 68$ & & $39 / 68$ & & $39 / 68$ & & \\
\hline Isoflavones $(\mathrm{mg} / \mathrm{d})$ & 1.4 & 1.0 & $5 \cdot 1$ & 1.2 & 10.9 & $2 \cdot 3$ & $36 \cdot 0$ & $60 \cdot 1$ & \\
\hline Range & \multicolumn{2}{|c|}{$0-3.24$} & \multicolumn{2}{|c|}{$3.21-7.94$} & \multicolumn{2}{|c|}{$5 \cdot 95-16 \cdot 15$} & \multicolumn{2}{|c|}{$14 \cdot 20-610 \cdot 2$} & \\
\hline Energy $(\mathrm{kJ} / \mathrm{d})$ & 7632 & 3410 & 9581 & 3439 & 10251 & 5678 & 12334 & 6481 & 0.000 \\
\hline Fat (\% energy) & $29 \cdot 2$ & $10 \cdot 9$ & 34.0 & $12 \cdot 2$ & 35.8 & $12 \cdot 5$ & 37.4 & $12 \cdot 2$ & 0.000 \\
\hline BMI $\left(\mathrm{kg} / \mathrm{m}^{2}\right)$ & 23.5 & 3.6 & $23 \cdot 3$ & 3.3 & $23 \cdot 1$ & 3.3 & 23.0 & 3.2 & 0.794 \\
\hline WHR & 0.89 & 0.07 & 0.87 & 0.06 & 0.86 & 0.07 & 0.87 & 0.06 & 0.335 \\
\hline $\mathrm{SBP}(\mathrm{mmHg})$ & $125 \cdot 6$ & 19.5 & 123.7 & $18 \cdot 6$ & $125 \cdot 0$ & $18 \cdot 7$ & $125 \cdot 7$ & $18 \cdot 7$ & 0.847 \\
\hline $\mathrm{DBP}(\mathrm{mmHg})$ & $80 \cdot 7$ & 10.5 & 80.5 & $11 \cdot 1$ & $81 \cdot 0$ & 9.87 & $82 \cdot 4$ & $10 \cdot 7$ & 0.552 \\
\hline
\end{tabular}

WHR, waist:hip ratio; SBP, systolic blood pressure; DBP, diastolic blood pressure. ${ }^{*}$ For contrasting groups using ANOVA.

$(P=0.055)$. Similarly, there was a significant linear decreasing trend of carotid CCA-IMT in equol excretors in different quartiles of isoflavone intake $(P=0 \cdot 044)$. As shown in Table 3 , subjects in the first quartile of daily isoflavone intake had a significantly higher CCA-IMT than did those in the second, third and fourth quartiles $(P<0.05$ for all).

There were no significant trends towards blood lipids or IMT in non-excretors, and no significant differences were observed in blood lipids or IMT in non-excretors in the different quartiles of dietary isoflavone intake.

\section{Discussion}

In the present study, we demonstrated that equol excretors had a lower lipid profile and IMT than non-excretors, and the lipid-lowering and vascular health effects of isoflavones were limited to equol excretors.

Equol excretors on their usual diet in the present study represented $25.0 \%$ of the southern Chinese adults. The finding is consistent with the previously reported frequency of equol excretion in Chinese adults ${ }^{(12,16)}$, but lower than that reported in $\operatorname{Japan}^{(17)}$.

In our previous study ${ }^{(12)}$, we found a significantly higher percentage of equol excretion in Chinese adults who had consumed soya before urine collection than in those on their usual diet. However, in the present study, we did not observe a significantly higher excretion percentage of equol in Chinese adults across quartiles of soya isoflavone consumption, suggesting that usual dietary intake did not affect the equol excretion status. The half-lifes of daidzein and equol in the circulation are $5-8 \mathrm{~h}^{(18)}$. Then, using the first urine passed in the morning to check equol is liable to the induction of the dinner on the previous day, although in adults, the ability to produce equol does not appear to be easily altered by dietary means ${ }^{(19-21)}$.

Carotid artery stenosis is a subclinical marker of systemic atherosclerosis, including coronary artery diseases and cerebrovascular diseases. An increased carotid IMT is a stronger predictor of CHD and stroke ${ }^{(22,23)}$. A previous study by our group found that higher habitual soya food consumption was associated with decreased bifurcation IMT, plasma total cholesterol and LDL-cholesterol in middle-aged Chinese adults $^{(24)}$. Other studies have examined the association between habitual soya food intake and the risk of CVD, and yielded inconsistent results. The Shanghai Women's Health Study, including 64915 women and with a mean follow-up of 2.5 years, showed that covariate-adjusted CHD risk decreased by $75 \%$ in middle-aged women consuming a median soya protein of $1.99 \mathrm{~g} / \mathrm{d}$ when compared with those with a median intake of $0.47 \mathrm{~g} / \mathrm{d}^{(4)}$. Another 7 -year prospective study in Japan reported a marginally significantly inverse relationship between soya product intake and total mortality in 13355 males and 15724 females, respectively ${ }^{(25)}$. However, a report from the Japan Public Health Center-Based Study Cohort exhibited sex-specific relationships between soya and isoflavone intake and the risk of cerebral and myocardial infarctions ${ }^{(26)}$. High isoflavone intake was associated with a reduced risk of cerebral and myocardial infarctions in Japanese women. The risk reduction was pronounced for postmenopausal women, but no significant association of 
Table 3. Blood lipids and clinical characteristics across quartiles of soya isoflavone intakes in equol excretors or non-excretors (Mean values and standard deviations)

\begin{tabular}{|c|c|c|c|c|c|c|c|c|c|c|c|}
\hline & \multirow[b]{3}{*}{ Model } & \multicolumn{8}{|c|}{ Soya isoflavone intake } & \multirow[b]{3}{*}{$P \S$} & \multirow[b]{3}{*}{$P \|$} \\
\hline & & \multicolumn{2}{|c|}{1 (low) } & \multicolumn{2}{|c|}{2} & \multicolumn{2}{|c|}{3} & \multicolumn{2}{|c|}{4 (high) } & & \\
\hline & & Mean & SD & Mean & SD & Mean & SD & Mean & SD & & \\
\hline \multicolumn{12}{|c|}{ Equol excretors } \\
\hline \multirow[t]{2}{*}{ TC } & Model 1 & $5 \cdot 70$ & $0 \cdot 16$ & 5.58 & $0 \cdot 16$ & $5 \cdot 74$ & 0.16 & $5 \cdot 61$ & 0.16 & 0.883 & 0.886 \\
\hline & Model 2 & $5 \cdot 70$ & $0 \cdot 17$ & $5 \cdot 52$ & 0.16 & $5 \cdot 77$ & 0.16 & 5.69 & 0.17 & 0.759 & 0.670 \\
\hline \multirow[t]{2}{*}{ HDL-C } & Model 1 & $1.43^{*}$ & 0.08 & $1.46^{*}$ & 0.07 & $1.47^{*}$ & 0.07 & $1 \cdot 70$ & 0.07 & 0.038 & 0.016 \\
\hline & Model 2 & 1.43 & 0.08 & 1.44 & 0.08 & 1.48 & 0.08 & 1.71 & 0.08 & 0.055 & 0.017 \\
\hline \multirow[t]{2}{*}{ LDL-C } & Model 1 & 3.55 & 0.14 & 3.45 & 0.14 & 3.53 & 0.14 & 3.26 & 0.14 & 0.433 & 0.199 \\
\hline & Model 2 & 3.52 & 0.14 & 3.42 & 0.14 & 3.54 & 0.14 & $3 \cdot 31$ & 0.15 & 0.687 & 0.456 \\
\hline \multirow[t]{2}{*}{ TAGף } & Model 1 & 1.62 & 0.14 & 1.42 & 0.14 & 1.55 & 0.14 & 1.33 & 0.14 & 0.234 & 0.099 \\
\hline & Model 2 & 1.56 & 0.14 & 1.47 & 0.14 & 1.55 & 0.14 & $1 \cdot 33$ & 0.14 & 0.399 & 0.164 \\
\hline \multirow[t]{2}{*}{ CB-IMT } & Model 1 & 1.02 & 0.06 & 1.03 & 0.05 & 1.07 & 0.05 & 0.98 & 0.05 & 0.719 & 0.743 \\
\hline & Model 2 & 1.02 & 0.06 & 1.03 & 0.05 & 1.07 & 0.05 & 0.98 & 0.05 & 0.719 & 0.743 \\
\hline \multirow[t]{2}{*}{ CCA-IMT } & Model 1 & 0.68 & 0.04 & $0.58 \dagger$ & 0.02 & $0.56 \dagger$ & 0.03 & $0.55 \dagger$ & 0.03 & 0.030 & 0.032 \\
\hline & Model 2 & 0.68 & 0.04 & $0.57 \dagger$ & 0.03 & $0.56 \dagger$ & 0.03 & $0.56 \dagger$ & 0.04 & 0.046 & 0.044 \\
\hline \multicolumn{12}{|c|}{ Non-excretors } \\
\hline \multirow[t]{2}{*}{$\mathrm{TC}$} & Model 1 & $5 \cdot 70$ & $0 \cdot 10$ & $5 \cdot 74$ & $0 \cdot 10$ & $5 \cdot 78$ & 0.10 & $5 \cdot 76$ & 0.10 & 0.946 & 0.619 \\
\hline & Model 2 & $5 \cdot 70$ & $0 \cdot 10$ & $5 \cdot 73$ & 0.10 & $5 \cdot 77$ & 0.10 & $5 \cdot 76$ & 0.10 & 0.977 & 0.687 \\
\hline \multirow[t]{2}{*}{ HDL-C } & Model 1 & 1.47 & 0.04 & 1.47 & 0.04 & 1.49 & 0.04 & 1.50 & 0.04 & 0.902 & 0.483 \\
\hline & Model 2 & 1.48 & 0.04 & 1.47 & 0.04 & 1.49 & 0.04 & 1.50 & 0.04 & 0.933 & 0.646 \\
\hline \multirow[t]{2}{*}{ LDL-C } & Model 1 & 3.35 & 0.09 & 3.49 & 0.09 & 3.50 & 0.08 & 3.42 & 0.09 & 0.600 & 0.585 \\
\hline & Model 2 & 3.37 & 0.09 & 3.49 & 0.09 & 3.49 & 0.09 & 3.42 & 0.09 & 0.685 & 0.691 \\
\hline \multirow[t]{2}{*}{ TAGף } & Model 1 & 1.98 & $0 \cdot 18$ & 1.91 & 0.18 & 1.67 & 0.19 & 1.88 & 0.19 & 0.388 & 0.183 \\
\hline & Model 2 & 1.94 & 0.19 & 1.91 & 0.18 & 1.69 & 0.19 & 1.91 & 0.19 & 0.557 & 0.325 \\
\hline \multirow[t]{2}{*}{ CB-IMT } & Model 1 & 1.08 & 0.04 & 1.05 & 0.04 & 1.05 & 0.04 & 1.07 & 0.04 & 0.907 & 0.915 \\
\hline & Model 2 & 1.07 & 0.04 & 1.05 & 0.04 & 1.06 & 0.04 & 1.09 & 0.04 & 0.911 & 0.759 \\
\hline \multirow[t]{2}{*}{ CCA-IMT } & Model 1 & 0.68 & 0.03 & 0.65 & 0.03 & 0.62 & 0.03 & 0.61 & 0.03 & 0.492 & 0.135 \\
\hline & Model 2 & 0.67 & 0.04 & 0.65 & 0.03 & 0.62 & 0.03 & 0.61 & 0.03 & 0.596 & 0.084 \\
\hline
\end{tabular}

TC, total cholesterol; HDL-C, HDL-cholesterol; LDL-C, LDL-cholesterol; CB-IMT, intima-media thickness at the carotid bulb; CCA-IMT, intima-media thickness at the common carotid artery.

${ }^{*}$ Mean values were significantly different compared with group $4(P<0.05)$.

† Mean values were significantly different compared with group $1(P<0.05)$.

$\ddagger$ Model 1, unadjusted; model 2, adjusted for age, energy, fat (\% energy).

$\S P$ for differences between the quartiles.

$\| P$ for trend.

ๆ Log transformed.

the dietary intake of soya and isoflavones with cerebral or myocardial infarctions was present in men ${ }^{(26)}$. The inconsistent relationships between cardiovascular benefits and soya protein or isoflavones were postulated to be, at least partly, due to the ability to produce equol. Consistent with the hypothesis, we observed a favourable relationship between TAG, IMT and the equol phenotype among middle-aged Chinese adults, suggesting that the ability to produce equol might play a role in modulating cardiovascular risk factors.

The precise molecular mechanisms linking daidzein or its metabolite equol to cardiovascular health remain unclear. A likely explanation for the TAG reduction was that equol can activate PPAR, which leads to decreased TAG concentrations via increased fatty acid oxidation ${ }^{(27)}$. Equol has a greater antioxidant activity than the parent isoflavone compounds genistein and daidzein, and its antioxidant effects during J774 cell-mediated LDL modification are based on a down-regulation of $\mathrm{O}_{2}^{-\cdot}$ production that is achieved, at least in part, through inhibited NADPH oxidase activity ${ }^{(28)}$.

An objective of the present study was to determine whether an individual's ability to convert daidzein to equol enhanced the lipid-lowering effects of isoflavones. The study by Meyer et $a l .{ }^{(29)}$ was the first human intervention to investigate retrospectively the correlation between equol production and the lipid-lowering benefits of soya supplementation. It showed significant reductions in the lipid profiles of equol producers compared with equol non-producers. In the present study, when subjects were categorised as either equol or equol non-excretors, there were significant differences in their responses to the dietary isoflavone intake. We observed that the benefits of isoflavones on CVD were limited to equol excretors. The results of the present study showed that a higher daily intake of isoflavones was associated with higher HDL-cholesterol and lower IMT in equol excretors other than equol non-excretors. The present finding confirms the hypothesis by Watanabe et $a l .{ }^{(9)}$, who suggested that the inconsistency in results from trials investigating the effects of isoflavones may be related to the ability of individuals to convert the soya isoflavone daidzein into equol.

The major limitation of the present study is that we examined the equol metabolic phenotype on the individuals' usual diet. According to our previous study ${ }^{(12)}$, the prevalence of equol producers increased from 13.4 to $50 \%$ after a $3 \mathrm{~d}$ soya isoflavone challenge, which suggested that a proportion of subjects had bacteria capable of producing equol but the insufficient isoflavone intake before urine collection resulted in them being classified as equol 
non-producers. The misclassification would bias the findings. However, such misclassification grouped some equol producers into equol non-producers, attenuating the true differences in blood lipids or IMT between equol producers and non-producers, which means that we are likely to underestimate the effects of equol metabolic phenotype on cardiovascular risk factors. In addition, we determined equol status with the urinary equol: daidzein ratio but not the detectable value of equol in the urine, which will reduce the possibility of misclassification to a certain degree.

There are some further limitations of the present study. We did not have enough power to detect small differences in blood lipids due to the relatively small study size. The results of the present study should be confirmed in a larger sample. Due to the limitation of funding, only the right carotid IMT was measured. The bias from other dietary factors such as vegetables and fish, even the parent isoflavone compounds genistein and daidzein and other daidzein metabolites, O-desmethylangolensin, could not be excluded.

In conclusion, higher dietary isoflavone consumption is associated with lower serum TAG and CCA-IMT in middle-aged Chinese equol producers, but not in equol non-producers. The present findings suggest that the benefits of soya isoflavones in preventing CVD may be limited to individuals with a high intrinsic capacity to convert daidzein to equol.

\section{Acknowledgements}

The present study was supported by the National Natural Science Foundation of China (30872102). The authors wish to thank the volunteers who participated in the study. Y. C. and K. G. contributed equally to the data collection, analysis and the manuscript preparation. C. C., P. W. and B. Z. were responsible for the statistical analysis and revision of the manuscript. Q. Z. and F. M. contributed to the assay of the isoflavone metabolites. B. Z. and Y. S. were responsible for the conception and design of the study. None of the authors had any financial or personal conflict of interest.

\section{References}

1. Setchell KD (1998) Phytoestrogens: the biochemistry, physiology, and implications for human health of soy isoflavones. Am J Clin Nutr 68, 1333S-1346S.

2. Setchell KD, Clerici C, Lephart ED, et al. (2005) S-Equol, a potent ligand for estrogen receptor beta, is the exclusive enantiomeric form of the soy isoflavone metabolite produced by human intestinal bacterial flora. Am J Clin Nutr 81, 1072-1079.

3. Lichtenstein AH (1998) Soy protein, isoflavones and cardiovascular disease risk. J Nutr 128, 1589-1592.

4. Zhang X, Shu XO, Gao YT, et al. (2003) Soy food consumption is associated with lower risk of coronary heart disease in Chinese women. J Nutr 133, 2874-2878.

5. Zhan S \& Ho SC (2005) Meta-analysis of the effects of soy protein containing isoflavones on the lipid profile. $A m J$ Clin Nutr 81, 397-408.

6. Reynolds K, Chin A, Lees KA, et al. (2006) A meta-analysis of the effect of soy protein supplementation on serum lipids. Am J Cardiol 98, 633-640.
7. Sacks FM, Lichtenstein A, Van Horn L, et al. (2006) Soy protein, isoflavones, and cardiovascular health: an American Heart Association Science Advisory for professionals from the Nutrition Committee. Circulation 113, 1034-1044.

8. Weggemans RM \& Trautwein EA (2003) Relation between soy-associated isoflavones and LDL and HDL cholesterol concentrations in humans: a meta-analysis. Eur J Clin Nutr 57, 940-946.

9. Watanabe S, Yamaguchi M, Sobue T, et al. (1998) Pharmacokinetics of soybean isoflavones in plasma, urine and feces of men after ingestion of $60 \mathrm{~g}$ baked soybean powder (kinako). J Nutr 128, 1710-1715.

10. Song KB, Atkinson C, Frankenfeld CL, et al. (2006) Prevalence of daidzein-metabolizing phenotypes differs between Caucasian and Korean American women and girls. J Nutr 136, 1347-1351.

11. Karr SC, Lampe JW, Hutchins AM, et al. (1997) Urinary isoflavonoid excretion in humans is dose dependent at low to moderate levels of soy-protein consumption. Am J Clin Nutr 66, 46-51.

12. Guo K, Zhang B, Chen C, et al. (2010) Daidzein-metabolising phenotypes in relation to serum lipids and uric acid in adults in Guangzhou, China. Br J Nutr 104, 118-124.

13. Franke A, Morimoto Y, Yeh L, et al. (2006) Urinary isoflavonoids as a dietary compliance measure among premenopausal women. Asia Pac J Clin Nutr 15, 88-94.

14. Tseng M, Olufade T, Kurzer MS, et al. (2008) Food frequency questionnaires and overnight urines are valid indicators of daidzein and genistein intake in U.S. women relative to multiple 24-h urine samples. Nutr Cancer 60, 619-626.

15. Setchell KD \& Cole SJ (2006) Method of defining equol-producer status and its frequency among vegetarians. J Nutr 136, 2188-2193.

16. Liu B, Qin L, Liu A, et al. (2010) Prevalence of the equol-producer phenotype and its relationship with dietary isoflavone and serum lipids in healthy Chinese adults. J Epidemiol 20, 377-384.

17. Arai Y, Uehara M, Sato Y, et al. (2000) Comparison of isoflavones among dietary intake, plasma concentration and urinary excretion for accurate estimation of phytoestrogen intake. J Epidemiol 10, 127-135.

18. Setchell KD \& Cassidy A (1999) Dietary isoflavones: biological effects and relevance to human health. J Nutr 129, 758S-767S.

19. Frankenfeld CL, Atkinson C, Thomas WK, et al. (2005) High concordance of daidzein-metabolizing phenotypes in individuals measured 1 to 3 years apart. Br J Nutr 94, 873-876.

20. Lampe JW, Skor HE, Li S, et al. (2001) Wheat bran and soy protein feeding do not alter urinary excretion of the isoflavan equol in premenopausal women. J Nutr 131, 740-744.

21. Vedrine N, Mathey J, Morand C, et al. (2006) One-month exposure to soy isoflavones did not induce the ability to produce equol in postmenopausal women. Eur J Clin Nutr 60 , 1039-1045.

22. O'Leary DH, Polak JF, Kronmal RA, et al. (1999) Carotid-artery intima and media thickness as a risk factor for myocardial infarction and stroke in older adults. Cardiovascular Health Study Collaborative Research Group. $N$ Engl $J$ Med 340, 14-22.

23. Hollander M, Hak AE, Koudstaal PJ, et al. (2003) Comparison between measures of atherosclerosis and risk of stroke: the Rotterdam Study. Stroke 34, 2367-2372.

24. Zhang B, Chen YM, Huang LL, et al. (2008) Greater habitual soyfood consumption is associated with decreased carotid intima-media thickness and better plasma lipids in Chinese middle-aged adults. Atherosclerosis 198, 403-411. 
25. Nagata C, Takatsuka N \& Shimizu H (2002) Soy and fish oil intake and mortality in a Japanese community. Am J Epidemiol 156, 824-831.

26. Kokubo Y, Iso H, Ishihara J, et al. (2007) Association of dietary intake of soy, beans, and isoflavones with risk of cerebral and myocardial infarctions in Japanese populations - The Japan Public Health Center-Based (JPHC) Study Cohort I. Circulation 116, 2553-2562.

27. Ricketts ML, Moore DD, Banz WJ, et al. (2005) Molecular mechanisms of action of the soy isoflavones includes activation of promiscuous nuclear receptors. A review. I Nutr Biochem 16, 321-330.

28. Hwang J, Wang J, Morazzoni P, et al. (2003) The phytoestrogen equol increases nitric oxide availability by inhibiting superoxide production: an antioxidant mechanism for cellmediated LDL modification. Free Radic Biol Med 34, $1271-1282$.

29. Meyer BJ, Larkin TA, Owen AJ, et al. (2004) Limited lipidlowering effects of regular consumption of whole soybean foods. Ann Nutr Metab 48, 67-78. 